\title{
Outcomes of surgical aortic valve replacement in moderate risk patients: Implications for determination of equipoise in the transcatheter era
}

\author{
Sebastian A. Iturra, MD, Rakesh M. Suri, MD, DPhil, Kevin L. Greason, MD, John M. Stulak, MD, \\ Harold M. Burkhart, MD, Joseph A. Dearani, MD, and Hartzell V. Schaff, MD
}

\begin{abstract}
Objective: To determine the contemporary outcomes of surgical aortic valve replacement (SAVR) in a moderate surgical risk population.

Methods: We studied 502 consecutive adults who had undergone isolated SAVR from January 2002 to June 2011 for severe aortic valve stenosis with a Society of Thoracic Surgery predicted risk of mortality of $4 \%$ to $8 \%$. We included concomitant coronary artery bypass and aortic annular enlargement but not other concomitant procedures. The updated Valve Academic Research Consortium definitions were used, as appropriate.
\end{abstract}

\begin{abstract}
Results: The median age was 80 years (range, 49-96), 323 (64.3\%) had New York Heart Association class III-IV symptoms, and $101(20.1 \%)$ had undergone previous coronary artery bypass grafting. The mean predicted risk of mortality was $5.6 \%$. Concomitant coronary artery bypass grafting was performed in $270(53.8 \%)$. Re-exploration for bleeding occurred in $29(5.8 \%)$, stroke in $9(1.8 \%)$, and vascular complications in $2(0.4 \%)$. In the cohort, 14 early deaths $(2.8 \%)$ occurred. During follow-up (1174 days), 175 patients died. Using multivariate logistic regression analysis, the significant independent predictors of mid-term death included chronic pulmonary disease (hazard ratio, 2.00, 95\% confidence interval, $1.41-2.84 ; P<.001$ ), peripheral vascular disease (hazard ratio, $1.58 ; 95 \%$ confidence interval, $1.05-2.37 ; P=.029$ ), and atrial fibrillation (hazard ratio, 1.75; 95\% confidence interval, 1.16-2.65; $P=.008$ ).

Conclusions: SAVR in moderate-risk patients is currently performed with one half of the early predicted risk $(2.8 \%)$ and a low likelihood of complications, including a $1.8 \%$ incidence of stroke. Patients counseled for randomization to transcatheter aortic valve insertion should be informed of the excellent early to mid-term outcomes of SAVR, particularly those without pulmonary impairment, peripheral vascular disease, or atrial fibrillation. (J Thorac Cardiovasc Surg 2014;147:127-32)
\end{abstract}

Senile calcific aortic valve stenosis is the most frequent heart valve condition of elderly patients and has been associated with an excess mortality once symptoms appear. ${ }^{1-3}$ Surgical aortic valve replacement (SAVR) has been proved to improve symptoms and prolong survival, with very low morbidity and mortality. ${ }^{4}$ Recent randomized controlled trials have demonstrated that transcatheter aortic

From the Division of Cardiovascular Surgery, Mayo Clinic, Rochester, Minn.

The Division of Cardiovascular Surgery, Mayo Clinic (Rochester, Minn) funded the present study, including the statistical analysis.

Disclosures: The Division of Cardiovascular Surgery has a research grant titled "Randomized biological aortic valve replacement," funded equally by Edwards Lifesciences, St Jude Medical, and the Sorin Group. Dr Suri is the Principal Investigator for the PERCEVAL IDE trial funded by the Sorin Group. Dr Park serves on the advisory board for Thoratec and the Cleveland Clinic. None of the disclosures pertain to the present investigation. All other authors have nothing to disclose with regard to commercial support.

Read at the 93rd Annual Meeting of The American Association for Thoracic Surgery, Minneapolis, Minnesota, May 4-8, 2013.

Received for publication May 5, 2013; revisions received July 19, 2013; accepted for publication Aug 10, 2013; available ahead of print Oct 7, 2013.

Address for reprints: Rakesh M. Suri, MD, DPhil, Division of Cardiac Surgery, Mayo Clinic, 200 First St SW, Rochester, MN 55902 (E-mail: suri.rakesh@mayo.edu). 0022-5223/\$0.00

Published by Elsevier Inc. on behalf of The American Association for Thoracic Surgery

http://dx.doi.org/10.1016/j.jtcvs.2013.08.036 valve implantation (TAVR) offers similar rates of survival and symptom improvement to high-risk and inoperable patients at 2 years of follow-up. ${ }^{5}$ When considering therapy for patients with aortic stenosis (AS) at lower surgical risk, genuine uncertainty exists regarding the best treatment option, particularly for those at moderate risk of death after SAVR (Society of Thoracic Surgeons [STS] predicted risk of mortality [PROM], 4-8\%). Three randomized controlled trials are currently underway to ascertain the differences in outcomes after SAVR versus TAVR in moderate-risk patients: the Placement of Aortic Transcatheter Valve (PARTNER) IIA trial, studying the Edwards SAPIEN XT valve (Edwards Lifesciences, Irvine, Calif); Surgical Replacement and Transcatheter Aortic Valve Implantation (SURTAVI), using the Medtronic CoreValve System (Medtronic, Minneapolis, Minn); and the St Jude Portico trial (St Jude Medical, St Paul, Minn). The accurate prediction of perioperative morbidity and mortality in patients referred for TAVR evaluation remains challenging, however, because no risk model has been validated specifically for TAVR candidates and the currently used surgical scoring systems can either over- or underestimate the actual periprocedural risk. ${ }^{6}$ 


\section{Abbreviations and Acronyms \\ AS $=$ aortic stenosis \\ AVR $=$ aortic valve replacement \\ CABG = coronary artery bypass grafting \\ EuroSCORE $=$ European System for Cardiac \\ Operative Risk Evaluation \\ PROM = predicted risk of mortality \\ SAVR = surgical aortic valve replacement \\ STS = Society of Thoracic Surgeons \\ TAVR $=$ transcatheter aortic valve implantation}

We thus sought to test the hypothesis that the STS PROM would be capable of accurately predicting actual mortality in moderate-risk subgroups to help inform patients' decisions and better prepare cardiac care specialists to ascertain whether equipoise exists in the randomization of patients to TAVR or SAVR in current trials.

\section{METHODS}

\section{Study Design and Population}

The Mayo Clinic investigational review board approved the present study. Informed consent was waived according to the investigational review board specifications. From January 2002 to June 2011, 4499 SAVR procedures were performed at the Mayo Clinic (Rochester, Minn) to treat aortic valve stenosis. We included those with severe aortic valve stenosis (mean gradient $\geq 40 \mathrm{~mm} \mathrm{Hg}$ ) and an STS PROM of $4 \%$ to $8 \%$ (current version data set, 2.73 of the STS risk calculator). The records were excluded if they included multiple valve procedures or other major nonvalve-related operations, other than coronary artery bypass grafting $(\mathrm{CABG})$ and aortic annular enlargement.

\section{Clinical Data}

The Division of Cardiovascular Surgery database, patient medical records, follow-up questionnaires, and Social Security Death Index were reviewed for patient demographics, medical history, baseline symptoms, cardiac status, perioperative complications, readmission, and early and mid-term mortality. The variables evaluated during the study period included gender, age, left ventricular ejection fraction, STS PROM, body mass index, diabetes mellitus, hypertension, chronic lung disease, peripheral vascular disease, cerebrovascular disease, renal failure, atrial fibrillation, mitral regurgitation, coronary artery disease, previous $\mathrm{CABG}$, previous pacemaker, previous percutaneous coronary intervention, previous aortic valve balloonplasty, previous myocardial infarction, aortic valve gradient, New York Heart Association heart failure functional class, aortic annular enlargement, associated CABG, and urgent surgery. Data on these variables were collected in keeping with the standard definitions set forth by the STS as a part of the National Adult Cardiac Surgery Database. We used the clinical endpoint definitions recommended by the updated Valve Academic Research Consortium. ${ }^{7}$ We performed clinical follow-up examinations and sent surveys to all patients at regular intervals of 1,3 , and 5 years after surgery to ascertain their clinical status and postoperative complications.

\section{Statistical Analysis}

Continuous data are expressed as the mean \pm standard deviation or median and range. The determined values were compared with the early and mid-term mortality. Variables significant on univariate analysis were used during stepwise selection to create the final multivariate model. Statistical significance was considered at $P<.05$. Early operative mortality was defined as death occurring within 30 days of surgery or at any point during the index hospitalization.

\section{RESULTS}

A total of 502 patients who met the inclusion criteria were included in the present analysis. The baseline characteristics of the patients are listed in Table 1. The median patient age was 80 years (range, 49-96), and 277 patients $(55.2 \%)$ were men. The mean STS PROM was 5.6\% (standard deviation \pm 1.09 ). Hypertension was present in 415 patients $(82.7 \%)$, diabetes mellitus in $157(31.3 \%)$, chronic pulmonary disease in $119(23.7 \%)$, and renal failure, defined as a basal creatinine $\geq 2.0 \mathrm{mg} / \mathrm{dL}$, in 20 $(4.0 \%)$ ). Only 2 patients $(0.4 \%)$ were dialysis dependent preoperatively. Atrial fibrillation was present in 45 patients $(9.0 \%)$, previous myocardial infarction in $43(8.6 \%)$, previous percutaneous coronary intervention in $79(15.7 \%)$, and previous percutaneous aortic balloon-plasty in $2(0.4 \%)$. Of the 502 patients, $323(64.3 \%)$ presented with New York Heart Association class III-IV heart failure symptoms. The mean left ventricular ejection fraction was $60 \%$. Previous CABG had been performed in 101 patients $(20.1 \%)$.

Urgent surgery was required in 64 patients $(12.7 \%)$. The median crossclamp and cardiopulmonary bypass time was 60 and 82 minutes, respectively. Aortic valve bioprostheses were implanted in 477 patients $(95.0 \%)$. Concomitant CABG was necessary in 270 patients $(53.8 \%)$. Aortic annular enlargement with a pericardial patch was necessary in 25 patients $(5.0 \%)$. Red blood cell transfusion was required in 296 patients $(59.0 \%)$. The median mechanical ventilation and intensive care unit admission duration was 13.5 hours (range, 3-1092) and 28 hours (range, 5-849), respectively.

The frequencies of early complications are listed in Table 2. These included re-exploration for bleeding in 29 $(5.8 \%)$, atrial fibrillation or flutter in $207(41.2 \%)$, perioperative myocardial infarction in $2(0.4 \%)$, stroke in 9 $(1.8 \%)$, acute kidney injury stage II in $23(4.6 \%)$, new-onset dialysis in $11(2.1 \%)$, pneumonia in 20 $(4.0 \%)$, major vascular complications in $2(0.4 \%$; lower extremity ischemia secondary to the use of a femoral intra-aortic balloon pump), and deep sternal infection in 1 patient $(0.2 \%)$.

There were $14(2.8 \%)$ early deaths, of which $9(64.3 \%)$ were cardiac related. Three were immediate procedural mortalities according to the updated Valve Academic Research Consortium criteria. Of the 232 patients who had undergone isolated aortic valve replacement, 6 $(2.58 \%)$ died compared with 8 of $270(2.96 \%)$ who undergone associated CABG $(P=.798)$. We were unable to identify univariate predictors of early death, including previous $\mathrm{CABG}$, concomitant $\mathrm{CABG}$, and STS PROM 
TABLE 1. Baseline characteristics $(n=502)$

\begin{tabular}{|c|c|}
\hline Characteristic & Value \\
\hline Median age (y) & $80(49-96)$ \\
\hline Male gender & $277(55.2)$ \\
\hline Mean STS PROM (\%) & $5.6(\mathrm{SD} \pm 1.09)$ \\
\hline Mean body mass index $\left(\mathrm{kg} / \mathrm{m}^{2}\right)$ & $28(\mathrm{SD} \pm 6.05)$ \\
\hline \multicolumn{2}{|l|}{ NYHA class } \\
\hline I or II & $179(35.7)$ \\
\hline III or IV & $323(64.3)$ \\
\hline \multicolumn{2}{|l|}{ Type 2 diabetes mellitus } \\
\hline Total & $157(31.3)$ \\
\hline Insulin dependent & $53(10.6)$ \\
\hline Hypertension & $415(82.7)$ \\
\hline Cerebral vascular disease & $125(24.9)$ \\
\hline Peripheral vascular disease & $77(15.3)$ \\
\hline \multicolumn{2}{|l|}{ Chronic lung disease } \\
\hline Mild & $70(14.0)$ \\
\hline Moderate & $14(2.8)$ \\
\hline Severe & $35(7.0)$ \\
\hline Creatinine level $\geq 2.0 \mathrm{mg} / \mathrm{dL}$ & $20(4.0)$ \\
\hline Mean aortic valve gradient $(\mathrm{mm} \mathrm{Hg})$ & $51(40-112)$ \\
\hline \multicolumn{2}{|l|}{ Aortic regurgitation } \\
\hline Trivial or mild & $325(64.7)$ \\
\hline Moderate & $72(14.3)$ \\
\hline Severe & $5(1)$ \\
\hline \multicolumn{2}{|l|}{ Mitral regurgitation } \\
\hline Trivial or mild & $381(75.9)$ \\
\hline Moderate & $83(16.5)$ \\
\hline Severe & $2(0.4)$ \\
\hline Median LVEF (\%) & $63(13-84)$ \\
\hline Atrial fibrillation & $45(9.0)$ \\
\hline Coronary artery disease & $399(79.5)$ \\
\hline Permanent pacemaker & $26(5.2)$ \\
\hline Previous CABG & $101(20.1)$ \\
\hline Previous myocardial infarction & $43(8.6)$ \\
\hline Previous coronary percutaneous intervention & 79 (15.7) \\
\hline Previous percutaneous aortic balloon-plasty & $2(0.4)$ \\
\hline
\end{tabular}

(Table 3). We also combined the adverse outcomes of death plus stroke and renal failure and reoperation secondary to bleeding, and found no significant association with any of the previous variables analyzed (Table 3 ).

The median postoperative length of stay was 7 days (range, 3-70). Discharge to home was obtained for 328 $(65.3 \%)$, and the remaining patients required some type of assistance (extended care or a nursing home). Finally, 41 patients $(8.2 \%)$ required readmission within 30 days of surgery.

During the mid-term follow-up period of 1174 days, 175 patients died (out of hospital and $>30$ days after surgery). Overall survival at 2, 4, and 6 years was $87.86 \%$ (range, $84.79 \%-91.04 \%$ ), $74.75 \%$ (range, $70.46 \%-79.29 \%$ ), and $59.15 \%$ (range, $53.57 \%-65.32 \%$ ), respectively.
TABLE 2. Early postoperative complications $(n=502$ patients $)$

\begin{tabular}{lc}
\multicolumn{1}{c}{ Complication } & $\mathbf{n}(\%)$ \\
\hline Transfusion of red blood cells & $296(59.0)$ \\
$\quad \geq 2$ U & $228(45.4)$ \\
Atrial fibrillation or flutter & $207(41.2)$ \\
Reoperation for bleeding & $29(5.8)$ \\
Pneumonia & $20(4.0)$ \\
Acute kidney injury, stage II & $23(4.6)$ \\
Dialysis & $11(2.2)$ \\
Permanent pacemaker & $10(1.9)$ \\
Stroke & $9(1.8)$ \\
Perioperative myocardial infarction & $2(0.4)$ \\
Major vascular complications & $2(0.4)$ \\
Deep sternal infection & $1(0.2)$ \\
Endocarditis & 0 \\
\hline
\end{tabular}

On multivariate logistic regression analysis, the significant predictors of mid-term death included chronic pulmonary disease (hazard ratio, 2.00; 95\% confidence interval, $1.41-2.84 ; P<.001$ ), peripheral vascular disease (hazard ratio, $1.58 ; 95 \%$ confidence interval, $1.05-2.37 ; P=.029$ ), and atrial fibrillation (hazard ratio, $1.75 ; 95 \%$ confidence interval, 1.16-2.65; $P=.008$; Table 4). Compared with an age- and gender-matched general population group without AS, no statistically significant difference was found in late survival was identified (Figure 1).

TABLE 3. Univariate logistic regression models predicting early death

\begin{tabular}{lcc}
\hline \multicolumn{1}{c}{ Variable } & OR $(\mathbf{9 5} \% \mathbf{C I})$ & $\boldsymbol{P}$ value \\
\hline Gender & $2.27(0.75-6.86)$ & .148 \\
Age & $1.01(0.94-1.09)$ & .79 \\
LVEF & $1.01(0.96-1.06)$ & .689 \\
STS PROM & $1.07(0.66-1.73)$ & .774 \\
Body mass index & $0.93(0.83-1.03)$ & .147 \\
Diabetes & $0.59(0.16-2.15)$ & .425 \\
Hypertension & $0.76(0.21-2.79)$ & .682 \\
Chronic pulmonary disease & $1.89(0.42-8.85)$ & .408 \\
Peripheral vascular disease & $1.53(0.42-5.6)$ & .524 \\
Cerebral vascular disease & $1.7(0.56-5.18)$ & .348 \\
Renal failure & 0 & $\mathrm{NA}$ \\
Atrial fibrillation & 0 & $\mathrm{NA}$ \\
Mitral regurgitation & $1.36(0.37-5.04)$ & .648 \\
Previous pacemaker & $0.7(0.09-5.58)$ & .738 \\
Previous percutaneous coronary intervention & $2.03(0.6-6.93)$ & .257 \\
Previous CABG & $0.3(0.04-2.31)$ & .247 \\
Previous myocardial infarction & $1.92(0.59-6.28)$ & .278 \\
Aortic gradient & $0.99(0.94-1.04)$ & .651 \\
Chronic heart failure & $1.19(0.33-4.35)$ & .793 \\
Annular enlargement & $3.1(0.64-15.01)$ & .159 \\
Associated CABG & $1.15(0.39-3.36)$ & .798 \\
Urgent surgery & $0.52(0.07-4.04)$ & .531 \\
\hline$O R$, Ods & &
\end{tabular}

$O R$, Odds ratio; $C I$, confidence interval; $L V E F$, left ventricular ejection fraction; STS PROM, Society of Thoracic Surgeons predicted risk of mortality; $N A$, not applicable; $C A B G$, coronary artery bypass grafting. 
TABLE 4. Cox regression model predicting mid-term mortality

\begin{tabular}{|c|c|c|c|c|}
\hline \multirow[b]{2}{*}{ Variable } & \multicolumn{2}{|c|}{ Univariate } & \multicolumn{2}{|c|}{ Multivariate } \\
\hline & HR $(95 \%$ CI $)$ & $P$ value & HR $(95 \%$ CI $)$ & $P$ value $>$ chi-square \\
\hline Chronic pulmonary disease & $1.74(1.27-2.39)$ & $<.001$ & $2.00(1.41-2.84)$ & $<.001$ \\
\hline Peripheral vascular disease & $1.62(1.11-2.37)$ & .013 & $1.58(1.05-2.37)$ & .029 \\
\hline Atrial fibrillation & $1.67(1.11-2.53)$ & .014 & $1.75(1.16-2.65)$ & .008 \\
\hline
\end{tabular}

$H R$, Hazard ratio; $C I$, confidence interval; $P r$, probability.

\section{DISCUSSION}

In those with severe aortic valve stenosis who are at very high risk of SAVR or are considered surgically "inoperable," randomized prospective trials and observational series have reported improved survival after TAVR compared with medical therapy. ${ }^{8}$ Furthermore, TAVR has been shown to be as safe and effective as SAVR in those with high surgical risk. ${ }^{5}$ Despite the rapid expansion of information addressing these 2 subsets, however, a paucity of data is available specifically examining those at intermediate risk for SAVR with an STS PROM of $4 \%$ to $8 \%$ currently being considered for randomization in the PARTNER II, SURTAVI, and PORTICO studies worldwide. We thus sought to determine the contemporary outcomes of SAVR, adhering to criteria used in determining the candidacy for the PARTNER IIA trial to assist clinicians in ascertaining and conveying the results of SAVR to patients in the current era. ${ }^{9}$ We found that SAVR with or without combined CABG can be performed with approximately one half the early mortality predicted by the current version (data set 2.73) of the STS risk calculator. Surgical excision of the senescent native aortic valve, followed prosthetic valve replacement carried a very low risk of complications, including a $1.8 \%$ incidence of stroke and $<1 \%$ risk of major vascular complications.

Debate has been ongoing regarding the most accurate risk determination algorithm to assist cardiac care professionals with stratification of patients into various treatment modalities. ${ }^{10,11}$ Previous studies have demonstrated that the assessment of periprocedural risk using a risk calculator alone might not be completely reliable, with a tendency toward an overestimation of liability in higher risk patients. A previous report from our group concluded that the European System for Cardiac Operative Risk Evaluation (EuroSCORE) should not be used to determine operability for isolated SAVR owing to its tendency to overestimate mortality in the low-, intermediate-, and high-risk subgroups. ${ }^{12}$ Although the STS PROM is currently the most widely used system, it predicted nearly double the observed mortality in our present analysis. Current models could be improved by the addition of specific clinical and anatomic variables that affect mortality. For example, the presence of a porcelain aorta and frailty are important factors not included in either risk model but routinely considered during patient evaluation. Similar attempts to compare actual SAVR and TAVR mortality using the EuroSCORE and STS PROM in a high-risk population ${ }^{13}$ also showed better surgical results than that predicted using the currently available preoperative risk stratification models.

Four studies have been published comparing TAVR and SAVR in moderate- and/or low-risk patients. ${ }^{14-17} \mathrm{~A}$ nonrandomized study by Wenaweser and colleagues ${ }^{14}$ described the use of TAVR in low-risk (STS score, $<3 \%$ ), intermediate-risk (STS score, 3\%-8\%), and high-risk (STS score, $>8 \%$ ) patients, with all-cause mortality at 30 days of $2.4 \%, 3.9 \%$, and $14.9 \%(P<.001)$ and at 1 year of $10.1 \%, 16.1 \%$, and $34.5 \%(P<.0003)$, respectively. They concluded that candidacy for TAVR should not be guided by fulfillment of absolute risk score criteria but by the opinion of an interdisciplinary heart team after the clinical evaluation. The Italian Observational Study of Appropriateness, Efficacy, and Effectiveness of AVRTAVI Procedures for the Treatment of Severe Symptomatic Aortic Stenosis (OBSERVANT) research group studied a low- to moderate-risk matched population undergoing aortic valve replacement (mean logistic EuroSCORE, $9.4 \% \pm 10.4 \%$ for SAVR and $8.9 \% \pm 9.5 \%$ for TAVR). They reported a 30-day mortality of $3.8 \%$ for both groups. In their study, SAVR was associated with a greater risk of blood transfusion, and TAVR resulted in a significantly increased rate of vascular injury, permanent atrioventricular block, and residual aortic valve regurgitation. ${ }^{15}$ Another Italian study by Latib and colleagues ${ }^{16}$ identified 182 consecutive patients who had undergone TAVR, comparing them against 111 moderate- to high-risk historical SAVR case controls. Using propensity score matching groups, TAVR was associated with more frequent vascular complications $(33.3 \%$ vs $0.9 \%, P<.001)$, and acute kidney injury was more frequent after SAVR $(8.1 \%$ vs $26.1 \%, P<.001)$. The rate of all-cause mortality in both groups was $1.8 \%$ at 30 days $(P=1.00)$ and $6.4 \%$ and $8.1 \%$, respectively, at 1 year $(P=.80)$. The incidence of cerebrovascular events was similar in the 2 groups $(4.6 \%$ vs $9.1 \%, P=.19)$ at 1 year. ${ }^{16}$ The Danish Prospective Randomized Trial of Transapical Transcatheter Aortic Valve Implantation Versus Surgical Aortic Valve Replacement in Operable Elderly Patients With Aortic Stenosis (STACCATO) trial 


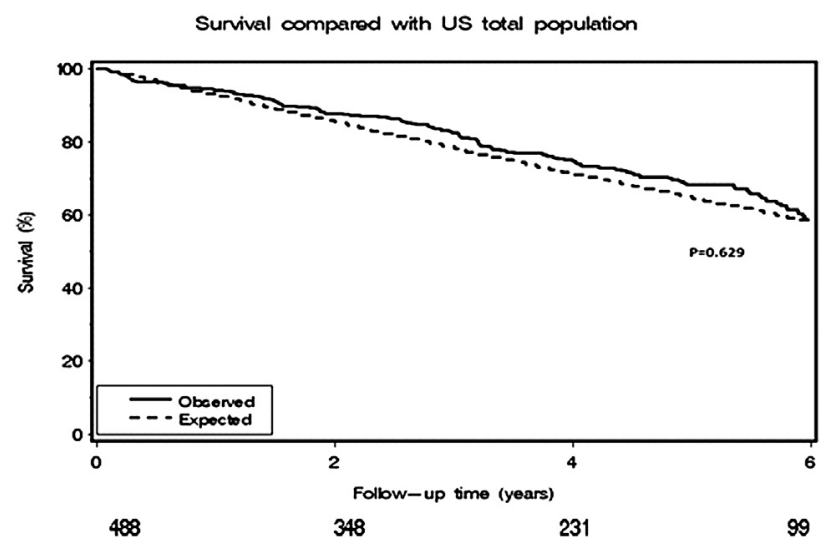

FIGURE 1. Kaplan-Meier late survival curve compared with survival of expected age- and gender-matched US population.

randomized elderly operable patients to TAVR and SAVR groups. However, the Data Safety Monitoring Board prematurely terminated the study after the enrollment of 70 patients because of adverse events: 2 deaths, 2 strokes, 1 case of renal failure requiring dialysis in the TAVR group and 1 stroke in the SAVR group $(P=.07)$. In the TAVR group, 1 patient required conversion to SAVR because of an abnormally positioned heart and 4 patients underwent reoperation with open heart surgery because of annulus rupture in 1 , severe paravalvular leakage in 2 , and blockage of the left coronary artery in $1 .{ }^{17}$

A large contemporary Italian study sought to determine the risk factors for mortality during SAVR and found that advanced heart failure, severe pulmonary hypertension, severe chronic obstructive pulmonary disease, and central neurologic dysfunction were independent predictors of early mortality. ${ }^{18}$ During mid-term follow-up, a low ejection fraction and advanced age were adverse prognostic factors. ${ }^{18}$ We did not discover any specific significant predictors of mortality in the early postoperative period in our present study. We did identify significant independent predictors of death, including chronic pulmonary disease, peripheral vascular disease, and atrial fibrillation. Our group has previously described the adverse effect of chronic obstructive lung disease as an independent risk factor for long-term mortality after SAVR. ${ }^{19}$

Another important finding to highlight is that SAVR was capable of restoring survival to that expected for an age- and gender-matched US population sample, demonstrating the important benefit of SAVR in the treatment of moderate-risk patients with severe aortic valve stenosis.

Finally, we acknowledge that the surgical outcomes from different hospitals could vary and that this could have been a limitation of the present study. This bias could be attributed to the complexity of the cases and the volume of SAVR in each respective center. Thus, we believe the heart team approach is vital to be able to determine the local data to give the best treatment option to patients with AS in a moderate-risk population in the current transcatheter era.

\section{Study Limitations}

We acknowledge that the surgical outcomes will vary between sites nationally and internationally. Despite this, our outcomes were likely similar to those of other transcatheter investigational sites currently participating in the Edwards PARTNER II, Medtronic SURTAVI, and St Jude Portico trials. The ultimate decisions regarding the patient treatment strategy should be made on a center-by-center basis according to the center- and team-based clinical experience and outcomes.

\section{CONCLUSIONS}

SAVR with or without combined CABG for severe AS in moderate-risk patients can be performed with approximately one half the early mortality predicted by the currently used risk models in North America. Surgical aortic valve excision with prosthetic replacement has a very low risk of complications, including a $1.8 \%$ incidence of stroke and $<1 \%$ likelihood of vascular complications. Patients being counseled for potential randomization to TAVR should be informed of the excellent early to mid-term outcomes of SAVR for those without pulmonary disease or advanced heart failure symptoms.

\section{References}

1. Nkomo VT, Gardin JM, Skelton TN, Gottdiener JS, Scott CG, EnriquezSarano M. Burden of valvular heart diseases: a population-based study. Lancet 2006;368:1005-11

2. Ross J, Braunwald E. Aortic stenosis. Circulation. 1968;38:61-7.

3. Turina J, Hess O, Sepulcri F, Krayenbuehl HP. Spontaneous course of aortic valve disease. Eur Heart J. 1987;8:471-83

4. Schwarz F, Baumann P, Manthey J, Hoffmann M, Schuler G, Mehmel HC, et al. The effect of aortic valve replacement on survival. Circulation. 1982;66: 1105-10.

5. Kodali SK, Williams MR, Smith CR, Svensson LG, Webb JG, Makkar RR, et al. Two-year outcomes after transcatheter or surgical aortic-valve replacement. N Engl J Med. 2012;366:1667-95

6. Piazza N, Wenaweser P, van Gameren M, Pilgrim T, Tzikas A, Otten A, et al. Relationship between the logistic EuroSCORE and the Society of Thoracic Surgeons Predicted Risk of Mortality score in patients implanted with the CoreValve ReValving system-a Bern-Rotterdam study. Am Heart J. 2010; 159:323-9.

7. Kappetein AP, Head SJ, Genereux P, Piazza N, van Mieghem NM, Blackstone EH, et al. Updated standardized endpoint definitions for transcatheter aortic valve implantation: the Valve Academic Research Consortium-2 consensus document. J Thorac Cardiovasc Surg. 2013;145:6-23.

8. Leon MB, Smith CR, Mack M, Miller DC, Moses JW, Svensson LG, et al Transcatheter aortic-valve implantation for aortic stenosis in patients who cannot undergo surgery. N Engl J Med. 2010;363:1597-607.

9. Brown JM, O’Brien SM, Wu C, Sikora JAH, Griffith BP, Gammie JS. Isolated aortic valve replacement in North America comprising 108,687 patients in 10 years: changes in risk, valve types, and outcomes in the Society of Thoracic Surgeons National Database. J Thorac Cardiovasc Surg. 2009; 137:82-90.

10. Wendt D, Osswald BR, Kayser K, Thielmann M, Tossios P, Massoudy P, et al Society of Thoracic Surgeons score is superior to the EuroSCORE determining mortality in high risk patients undergoing isolated aortic valve replacement Ann Thorac Surg. 2009;88:468-75. 
11. Richarson M, Howell N, Freemantle N, Bridgewater B, Pagano D. Prediction of in-hospital death following aortic valve replacement: a new accurate model. Eur J Cardiothorac Surg. 2012;43:704-8.

12. Brown ML, Schaff HV, Sarano ME, Li Z, Sundt TM, Dearani JA, et al. Is the European System for Cardiac Operative Risk Evaluation model valid for estimating the operative risk of patients considered for percutaneous aortic valve replacement? J Thorac Cardiovasc Surg. 2008;136:566-71.

13. Grossi EA, Schwartz CF, Yu PJ, Jorde UP, Crooke GA, Grau JB, et al. High-risk aortic valve replacement: are the outcomes as bad as predicted? Ann Thorac Surg. 2008:85:102-6.

14. Wenaweser P, Stortecky S, Schwander S, Heg D, Huber C, Pilgrim T, et al. Clinical outcomes of patients with estimated low or intermediate surgical risk undergoing transcatheter aortic valve implantation. Eur Heart J. 2013;34: 1894-905

15. D'Errigo P, Barbanti M, Ranucci M, Onorati F, Covello RD, Rosato S, et al. Transcatheter aortic valve implantation versus surgical aortic valve replacement for severe aortic stenosis: results from an intermediate risk propensity-matched population of the Italian OBSERVANT study. Int J Cardiol. 2013;167:1945-52.

16. Latib A, Maisano F, Bertoldi L, Giacomini A, Shannon J, Cioni M, et al. Transcatheter vs surgical aortic valve replacement in intermediate-surgical-risk patients with aortic stenosis: a propensity score-matched case-control study. Am Heart J. 2012;164:910-7.

17. Nielsen HH, Klaaborg KE, Nissen H, Terp K, Mortensen PE, Kjeldsen BJ, et al. A prospective, randomized trial of transapical transcatheter aortic valve implantation vs. surgical aortic valve: the STACCATO trial. EuroIntervention. 2012;8:383-9

18. Eusanio MD, Fortuna D, De Palma R, Dell'Amore A, Lamarra M, Contini GA, et al. Aortic valve replacement: results and predictors of mortality from a contemporary series of 2256 patients. J Thorac Cardiovasc Surg. 2011;141:940-7.

19. Spoon DB, Orzulak TA, Edell ES, Li Z, Nishimura RA. Risk of aortic valve replacement in patients with aortic stenosis and chronic obstructive pulmonary disease. J Heart Valve Dis. 2012;21:314-9.

\section{Discussion}

Dr Kenton J. Zehr (Temple, Tex). To the AATS Program Committee, moderators, and the Mayo Clinic group:

Thank you for the opportunity to discuss this report and the privilege of reviewing the full study before the meeting.

Dr Iturra and the Mayo Clinic group have presented a unique group of primarily elderly intermediate-risk group patients undergoing aortic valve replacement (AVR) or AVR plus CABG during the recent decade. Despite an STS risk score of $4 \%$ to $8 \%$, they observed mortality of $2.8 \%$. One remembers back to a report from the same institution presented by Drs Dwight McGoon and Emerson Moffit for the first 100 consecutive AVRs with a StarrEdwards valve without a single mortality. Good therapies find their way. This is another report attesting to the safety and efficacy of SAVR.

This study raises the question of the presumed equipoise to TAVR in this group of intermediate-risk patients, and I would rhetorically ask: "where is the evidence for equipoise?" You have seen the low rates of complications in this presented series, with a postoperative length of stay of 7 days and an $8.2 \%$ readmission rate at 30 days. Most of us can agree that the PARTNER trial strongly confirmed the benefits of TAVR in inoperable patients and suggested equipoise for high-risk surgical patients, but it came at a significant price. If one reviews the briefing for the Food and Drug Administration regarding the experience with the SAPIEN valve in the nonoperative cohort, there were 8 device malfunctions, 4 patients required an additional valve, and 7 patients never received a valve because of access issues and were excluded from the study. In the high-risk cohort study in the PARTNER trial, 4 intraprocedural deaths occurred, 3 in the TAVR group and 1 in the AVR group. Also, in 16 patients, the procedure was aborted or converted to AVR because of intraprocedural findings, 7 patients required $>1$ valve $(2$ because of embolization and 5 because of paravalvular leak), and 5 additional valve embolizations occurred. Cardiac mortality was the same in both groups, but the stroke rate was double. In the Danish STACCATO trial, the study was terminated.

Dr Woo. Could we ask you to ask the questions, we are short on time.

Dr Zehr. I have just 1 question.

I want to present a little bit of our recent data. We started using TAVR, and we have treated 34 patients within 8 months. We have had no intraprocedural deaths, but we have had 3 deaths within 30 days. They also come at a price: a few minor vascular complications, 1 stroke, and 2 patients who required median sternotomy for tamponade. So, we recognize the value of TAVR, but we believe it is a real operation with real complications and in no way have we concluded that current equipoise exists for intermediate-risk patients.

So, I have 1 question. How is your group addressing the pressure to perform TAVR in these intermediate-risk patients in lieu of your results and the current recommendations from the Food and Drug Administration and Centers for Medicare \& Medicaid Services?

Thank you very much.

Dr Iturra. Thank you for your comments and question.

The clinical records and imaging studies of each patient being considered for either Food and Drug Administration transcatheter trials or commercially available TAVR devices are discussed by a multidisciplinary heart team at the Mayo Clinic. When a patient is being considered for randomization, we use the currently available data and solicit the opinions of all heart team members to arrive at a determination of whether equipoise exists. If so, we provide full disclosure of the indications, risks, benefits, and alternatives of SAVR versus transcatheter valve therapy to each patient to allow them to make an informed decision. 